\title{
A CLASS OF TORUS MANIFOLDS WITH NONCONVEX ORBIT SPACE
}

\author{
MAINAK PODDAR AND SOUMEN SARKAR
}

(Communicated by Daniel Ruberman)

\begin{abstract}
We study a class of smooth torus manifolds whose orbit space has the combinatorial structure of a simple polytope with holes. We construct moment angle manifolds for such polytopes with holes and use them to prove that the associated torus manifolds admit stable almost complex structure. We give a combinatorial formula for the Hirzebruch $\chi_{y}$ genus of these torus manifolds. We show that they have (invariant) almost complex structure if they admit positive omniorientation. We give examples of almost complex manifolds that do not admit a complex structure. When the dimension is four, we calculate the homology groups and describe a method for computing the cohomology ring.
\end{abstract}

\section{INTRODUCTION}

The moment polytope of the Hamiltonian action of the real torus $\mathbb{T}^{n}$ on a smooth projective toric variety (toric manifold) may be identified with the orbit space of the action. The moment polytope (Delzant polytope) is rather rigid with severe integrality constraints; see Definition 2.1.1 of [Sil01]. In 1991 Davis and Januszkiewicz DJ91 introduced a generalization of toric manifolds, now known as quasitoric manifolds, which may be obtained as identification spaces of $\mathbb{T}^{n} \times P$ where $P$ is a simple $n$-dimensional polytope. In general these spaces do not have algebraic or invariant symplectic structure, but they still have many remarkable properties; see the survey BP02. In this article we study a class of even dimensional manifolds which may be obtained as identification space of $\mathbb{T}^{n} \times P$ where $P$ is not convex, but a simple polytope with holes which are also simple polytopes. In Mas99 and [HM03, Masuda and Hattori introduced the notion of torus manifold (see Definition 2.2. $)$. Our manifolds are a special class of torus manifolds. As in the case of quasitoric manifolds, the torus action on our manifolds is locally standard, i.e. locally equivalent to the natural action, up to automorphism, of $U(1)^{n}$ on $\mathbb{C}^{n}$.

We describe the combinatorial construction of these manifolds in section 2 However, these manifolds are also obtained by gluing quasitoric manifolds along deleted

Received by the editors September 28, 2011 and, in revised form, July 11, 2012 and July 18, 2013.

2010 Mathematics Subject Classification. Primary 57R17, 57R91.

Key words and phrases. Almost complex, symplectic, Hirzebruch genus, moment angle complex, torus action.

The first author was partially supported by the Proyecto de investigaciones grant from the Universidad de los Andes.

The second author was partially supported by the National Research Foundation of Korea (NRF) grant funded by the Korean government (MEST) (No. 2012-0000795). 
neighborhoods of principal torus orbits (Lemma 2.1). We refer to this as the fiber sum construction. It is a special case of a more general construction in GK98. This is used to endow the manifolds with smooth structure (Lemma 2.1), and in certain cases with almost complex structure (Theorem 5.1).

We realize each of our manifolds as the quotient of a submanifold of $\mathbb{C}^{m}$ by the free action of a compact torus in section 4. This may be viewed as a topological analogue of the construction of toric manifolds by symplectic reduction, or of the quotient construction of toric varieties. We use this to endow our manifolds with a stable complex structure (Lemma 5.1). Using it, we give a combinatorial formula for the $\chi_{y}$ genus of these manifolds (Theorem 5.3) following the work of Panov Pan01 in quasitoric case. The formula also follows from Lemma 5.1 and a more general result in [HM03.

Our manifolds admit almost complex structure if they admit a positive omniorientation (Lemma 5.2 and Theorem 5.1). Positive omniorientation is also a necessary condition if we require the almost complex structure to be $\mathbb{T}^{n}$-invariant.

These manifolds cannot admit an invariant symplectic structure (Lemma 5.3) or an invariant integrable complex structure (see IK12]) if the orbit space has at least one hole. It would be interesting to know if any of these torus manifolds admit a symplectic or complex structure. If the orbit space has one hole, then the manifold cannot be Kahler (Lemma 5.4). We give examples of almost complex manifolds that do not admit a complex structure in section 5.2

Much is known about the topological invariants of these manifolds from the works Mas99 and HM03. However as they have nontrivial homology in odd degrees (see Theorem 9.3 of [MP06]), the formula for the cohomology ring given in Corollary 7.8 of MP06] does not hold when the orbit space has holes. Even explicit formulas for their (co)homology groups are not known in general. In section 3, we give a combinatorial formula for the homology groups when the dimension is four. We also describe a method for computing the cohomology ring for the four dimensional manifolds.

\section{Construction And smooth structure}

2.1. Polytope with holes. A polytope is the convex hull of a finite set of points in $\mathbb{R}^{n}$. An $n$-dimensional polytope is said to be simple if every vertex is the intersection of exactly $n$ codimension one faces. Let $P_{0}$ be an $n$-dimensional simple polytope in $\mathbb{R}^{n}$. Let $P_{1}, P_{2}, \ldots, P_{s}$ be a disjoint collection of simple polytopes belonging to the interior of $P_{0}$. Let

$$
P=P_{0}-\bigcup_{k=1}^{s} P_{k}^{\circ} .
$$

We call $P$ an $n$-dimensional polytope with simple holes. The polytopes $P_{1}, P_{2}, \ldots$, $P_{s}$ are called holes of $P$. The faces of $P$ are the faces of $P_{k}, k=0, \ldots, s$.

2.2. Combinatorial construction. Let $P$ be an $n$-dimensional simple polytope with $s$ simple holes. Let $\mathcal{F}(P)=\left\{F_{1}, F_{2}, \ldots, F_{m}\right\}$ be the set of all codimension one faces (facets) of $P$. Note that $\mathcal{F}(P)=\bigcup_{k=0}^{s} \mathcal{F}\left(P_{k}\right)$. Also, if $F$ is a nonempty face of $P$ of codimension $k$, then $F$ is the intersection of a unique collection of $k$ facets of $P$. The following definition is a straightforward generalization of the notion of characteristic function for a simple polytope, which is a crucial concept for studying quasitoric manifolds [DJ91, BP02]. 

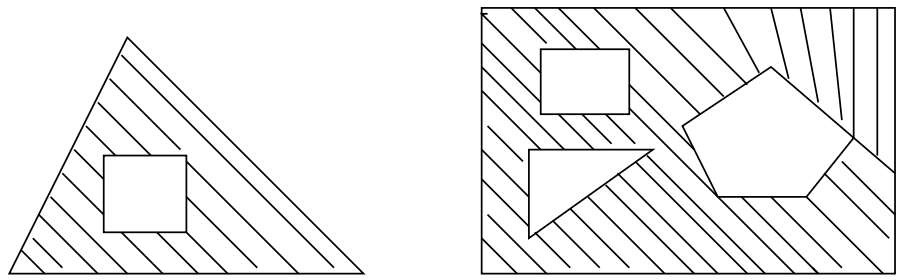

Figure 1. Polytopes with simple holes in $\mathbb{R}^{2}$.

Definition 2.1. A function $\lambda: \mathcal{F}(P) \rightarrow \mathbb{Z}^{n}$ is called a characteristic function if it satisfies the following condition: Whenever $F=\bigcap_{j=1}^{k} F_{i_{j}}$ is an $(n-k)$-dimensional face of $P$, the span of the vectors $\lambda\left(F_{i_{1}}\right), \lambda\left(F_{i_{2}}\right), \ldots, \lambda\left(F_{i_{k}}\right)$ is a $k$-dimensional direct summand of $\mathbb{Z}^{n}$. We will denote $\lambda\left(F_{i}\right)$ by $\lambda_{i}$ for simplicity and call it the characteristic vector of $F_{i}$.

For any face $F=\bigcap_{j=1}^{k} F_{i_{j}}$ of $P$, let $N(F)$ be the submodule of $\mathbb{Z}^{n}$ generated by $\lambda_{i_{1}}, \ldots, \lambda_{i_{k}}$. The module $N(F)$ defines a sub-torus $G_{F}$ of $\mathbb{T}^{n}=\mathbb{Z}^{n} \otimes \mathbb{R} / \mathbb{Z}^{n}=\mathbb{R}^{n} / \mathbb{Z}^{n}$ as follows:

$$
G_{F}:=(N(F) \otimes \mathbb{R}) / N(F)
$$

Define an equivalence relation $\sim$ on the product space $\mathbb{T}^{n} \times P$ by

$$
(t, x) \sim(u, y) \text { if } x=y \text { and } u^{-1} t \in G_{F(x)}
$$

where $F(x)$ is the unique face of $P$ whose relative interior contains $x$.

We denote the quotient space as follows:

$$
M=M(P, \lambda):=\left(\mathbb{T}^{n} \times P\right) / \sim
$$

The space $M$ is a $2 n$-dimensional manifold. The proof of this is analogous to the quasitoric case DJ91. The $\mathbb{T}^{n}$ action on $\left(\mathbb{T}^{n} \times P\right)$ induces a natural effective action of $\mathbb{T}^{n}$ on $M$, which is locally standard (see [DJ91]). Let $\pi: M \rightarrow P$ be the projection or orbit map defined by $\pi([(t, x)])=x$.

Definition 2.2 ([HM03]). A closed, connected, oriented, smooth manifold $Y$ of dimension $2 n$ with an effective smooth action of $\mathbb{T}^{n}$ with nonempty fixed point set is called a torus manifold if a preferred orientation is given for each characteristic submanifold. A characteristic submanifold is, by definition, any codimension two closed connected submanifold of $Y$, which is fixed by some circle subgroup of $\mathbb{T}^{n}$ and contains at least one $\mathbb{T}^{n}$-fixed point.

In the case of $M$, the $\mathbb{T}^{n}$-fixed point set corresponds bijectively to the set of vertices of $P$. Observe that the spaces $X_{i}:=\pi^{-1}\left(F_{i}\right), i=1, \ldots, m$, are the characteristic submanifolds of $M$. Each $X_{i}$ is a 2(n-1)-dimensional quasitoric manifold. In section 2.4 we explain how the characteristic function $\lambda$ endows each $X_{i}$ with a preferred orientation. We say that $M$ is the torus manifold derived from the characteristic pair $(P, \lambda)$. 


\subsection{Fiber sum construction.}

Lemma 2.1. The torus manifold $M(P, \lambda)$ is smooth and orientable.

Proof. By induction it is sufficient to prove that $M(P, \lambda)$ has a smooth structure when $P$ is a polytope with one hole, that is, $P=P_{0}-P_{1}^{0}$. Let $\mathcal{F}\left(P_{0}\right)$ and $\mathcal{F}\left(P_{1}\right)$ be the set of facets of $P_{0}$ and $P_{1}$ respectively. The restrictions $\lambda_{0}$ and $\lambda_{1}$ of $\lambda$ on $\mathcal{F}\left(P_{0}\right)$ and $\mathcal{F}\left(P_{1}\right)$ are characteristic functions on $P_{0}$ and $P_{1}$ respectively. Let $M_{0}$ and $M_{1}$ be the quasitoric manifolds associated to the characteristic pairs $\left(P_{0}, \lambda_{0}\right)$ and $\left(P_{1}, \lambda_{1}\right)$ respectively. These manifolds, being quasitoric, have smooth structure.

Let $\pi_{k}: M_{k} \rightarrow P_{k}, k=0,1$ be the orbit maps. Fix points $x_{k} \in P_{k}^{\circ}$. Let

$$
L_{k}=\pi_{k}^{-1}\left(x_{k}\right) \text {. }
$$

Let $U_{k} \subset M_{k}$ be a $\mathbb{T}^{n}$ invariant neighborhood of $L_{k}$ such that

$$
B_{k}:=\pi_{k}\left(U_{k}\right) \subset P_{k}
$$

is diffeomorphic to an open ball in $\mathbb{R}^{n}$.

The quasitoric manifolds $M_{k}$ are orientable. An orientation on $M_{k}$ is determined by orientations on $\mathbb{R}^{n} \supset P_{k}$ and $\mathbb{T}^{n}$. Suppose $\mathbf{p}=\left(p_{1}, \ldots, p_{n}\right)$ and let $\mathbf{q}=$ $\left(q_{1}, \ldots, q_{n}\right)$ be the standard Cartesian and angular coordinates on $\mathbb{R}^{n}$ and $\mathbb{T}^{n}$ respectively. Then the orientation on $M_{k}$ corresponding to the ordering $\frac{\partial}{\partial p_{1}}, \frac{\partial}{\partial q_{1}}, \ldots$, $\frac{\partial}{\partial p_{n}}, \frac{\partial}{\partial q_{n}}$ will be assumed.

By (2.6) there exist equivariant orientation preserving diffeomorphisms

$$
f_{k}: U_{k} \rightarrow \mathbb{T}^{n} \times B
$$

where $B$ is the unit $n$-ball centered at the origin. Denote the punctured unit $n$-ball, $B-\{0\}$, by $B^{-}$. Let $|\cdot|$ be the Euclidean norm on $\mathbb{R}^{n}$. Define

$$
r:=|\mathbf{p}| \quad \text { and } \quad \Theta=\left(\theta_{1}, \ldots, \theta_{n}\right):=\frac{\mathbf{p}}{r} .
$$

The space $M(P, \lambda)$ can be obtained from $M_{0}-L_{0}$ and $M_{1}-L_{1}$ by identifying $U_{0}-L_{0}$ and $U_{1}-L_{1}$ as follows. Let $g: B^{-} \rightarrow B^{-}$be the orientation preserving involution,

$$
g(\mathbf{p})=\frac{1-r}{r}\left(p_{1}, \ldots, p_{n-1},-p_{n}\right) .
$$

In other words, $g(r, \Theta)=\left(1-r, \theta_{1}, \ldots, \theta_{n-1},-\theta_{n}\right)$.

Define

$$
h=f_{0}^{-1} \circ(I d \times g) \circ f_{1} .
$$

Identify $U_{0}-L_{0}$ with $U_{1}-L_{1}$ by the orientation preserving equivariant diffeomorphism $h$.

Remark 2.1. Note that the map $g$, as used in (2.10), radially inverts a deleted neighborhood $B_{1}^{-}$of the point $x_{1}$ in $P_{1}^{\circ}$ and reflects it about the hyperplane $p_{n}=$ 0 . Then the map $h$ identifies it to a deleted neighborhood of $x_{0}$ in $P_{0}^{\circ}$. Up to homeomorphism, we can widen the puncture at $x_{0}$, and fit $P_{1}-B_{1}$ into it and thus recover our picture of the orbit space of the glued manifold as the polytope with hole $P_{0}-P_{1}^{\circ}$. A smooth embedding of this orbit space into Euclidean space is described in section 4 . We do not know for sure if the smooth structure on the orbit space coincides with the smooth structure of $P$ coming from its given embedding in $\mathbb{R}^{n}$. 
Remark 2.2. We refer to the above gluing construction as fiber sum construction because of its similarity to the symplectic fiber sum construction (see Gro86, Gom95]). A more general fiber sum construction for spaces with torus action was introduced in GK98.

Remark 2.3. As we may observe from section 4 the exact formula for the gluing map $g$ is not important for the smooth structure.

Remark 2.4. The sign of the characteristic vectors do not affect the equivariant diffeomorphism type of $M$. This follows from similar observation for quasitoric manifolds; see [DJ91, BR01].

2.4. Omniorientation. We fix an orientation for $M(P, \lambda)$ as above by choosing standard orientations on $\mathbb{T}^{n}$ and $\mathbb{R}^{n}$. Also each characteristic submanifold $X_{i}$ is quasitoric and hence orientable.

Definition 2.3. An omniorientation is an assignment of orientation for $M(P, \lambda)$ as well as for each $X_{i}$. Given such an assignment, we say that $M(P, \lambda)$ is omnioriented.

Given the above choice of orientation for $M$, the characteristic function $\lambda$ determines a natural omniorientation on $M$ as follows: The characteristic vector $\lambda_{i}$ determines a fiberwise $S^{1}$ action on the normal bundle of $X_{i}$, corresponding to the isotropy group $G_{F_{i}}$. This equips the normal bundle with a complex structure and therefore an orientation. This, together with the orientation on $M$, induces an orientation on $X_{i}$. We will refer to this omniorientation as the characteristic omniorientation.

Consider an omniorientation on $M$. Let $v \in M$ be a fixed point of the $\mathbb{T}^{n}$ action (or corresponding vertex of $P$ ). If the orientation of $T_{v}(M)$ determined by the orientation on $M$ and the orientations of characteristic submanifolds containing $v$ coincide, then the $\operatorname{sign} \sigma(v)$ is defined to be 1 , otherwise $\sigma(v)$ is -1 .

Definition 2.4. An omniorientation is called positive if $\sigma(v)=1$ for each fixed point $v$.

For the characteristic omniorientation, the sign of a vertex $v$ may be computed as follows [BP02]. Suppose $v=F_{i_{1}} \cap \ldots \cap F_{i_{n}}$. To each codimension one face $F_{i_{k}}$ assign the unique edge $E_{k}$ such that $E_{k} \cap F_{i_{k}}=v$. Let $e_{k}$ be a vector along $E_{k}$ with origin at $v$. Order (rename) the $e_{k} \mathrm{~s}$ so that $e_{1}, \ldots, e_{n}$ is a positively oriented basis for $\mathbb{R}^{n}$. Consider the corresponding matrix $\Lambda_{(v)}=\left[\lambda_{i_{1}} \ldots \lambda_{i_{n}}\right]$. Then

$$
\sigma(v)=\operatorname{det} \Lambda_{(v)} .
$$

Remark 2.5. It is also evident that the oriented intersection number of the submanifolds $X_{i_{1}}, \ldots, X_{i_{n}}$ is $\sigma(v)$.

\section{Calculations in dimension four}

Let $\pi: M(P, \lambda) \rightarrow P$ be a 4 -dimensional torus manifold, where $P$ is a polytope with $s$ simple holes. We give a $C W$ structure on $M(P, \lambda)$ and compute the homology groups.

First assume that $P$ has only one hole. Then $P=P_{0}-P_{1}^{0}$, where $P_{0}$ and $P_{1}$ are simple 2-dimensional polytopes with vertices $\left\{v_{1}, \ldots, v_{l_{0}}\right\}$ and $\left\{u_{1}, \ldots, u_{l_{1}}\right\}$ respectively. Assume that $\operatorname{dist}\left(v_{1} u_{1}\right) \leq \operatorname{dist}\left(v_{1} u_{j}\right)$ for all $j=1, \ldots, l_{1}$. Let $E_{v_{i}}$ and $E_{u_{j}}$ be the edges of $P$ joining the vertices $\left\{v_{i}, v_{i+1}\right\}$ and $\left\{u_{j}, u_{j+1}\right\}$ respectively for 
$i=1, \ldots, l_{0} ; j=1, \ldots, l_{1}$. Here assume $v_{l_{0}+1}=v_{1}$ and $u_{l_{1}+1}=u_{1}$. Let $E_{v_{1} u_{1}}$ be the line segment joining $v_{1}$ and $u_{1}$.

We construct the $i$-th skeleton $X_{i}$ of $M(P, \lambda)$ as follows. Let $X_{0}=\left\{v_{1}, \ldots\right.$, $\left.v_{l_{0}-1}, u_{1}, \ldots, u_{l_{1}}\right\}$. Define

$$
\begin{aligned}
& e_{i}^{1}=\left(\{(1,1)\} \times E_{v_{i}}\right) / \sim \quad \text { for } i=1, \ldots, l_{0}-2 \\
& e_{l_{0}-1}^{1}=\left(\{(1,1)\} \times E_{v_{1} u_{1}}\right) / \sim \\
& e_{l_{0}+j-1}^{1}=\left(\{(1,1)\} \times E_{u_{j}}\right) / \sim \text { for } j=1, \ldots, l_{1} \\
& X_{1}=\bigcup_{i=1}^{l_{0}+l_{1}-1} \frac{1}{e_{i}^{1}} .
\end{aligned}
$$

A picture of the 1-skeleton for a polytope with one hole is given in Figure 2(a). Define

$$
\begin{array}{ll}
e_{i}^{2}=\left(\left(\mathbb{T}^{2} \times E_{v_{i}}\right) / \sim\right)-\overline{e_{i}^{1}} & \text { for } i=1, \ldots, l_{0}-2 \\
e_{l_{0}-1}^{2}=\left(\left(\{1\} \times S^{1} \times E_{v_{1} u_{1}}\right) / \sim\right)-\overline{e_{l_{0}}^{1}} & \\
e_{l_{0}}^{2}=\left(\left(S^{1} \times\{1\} \times E_{v_{1} u_{1}}\right) / \sim\right)-\overline{e_{l_{0}}^{1}} & \\
e_{l_{0}+j}^{2}=\left(\left(\mathbb{T}^{2} \times E_{u_{j}}\right) / \sim\right)-\overline{e_{l_{0}+j-1}^{1}} & \text { for } j=1, \ldots, l_{1} \\
X_{2}=\bigcup_{i=1}^{l_{0}+l_{1}} \overline{e_{i}^{2}} . &
\end{array}
$$

Define

$$
\begin{aligned}
& e^{3}=\left(\left(\mathbb{T}^{2} \times E_{v_{1} u_{1}}\right) / \sim\right)-\left(\overline{e_{l_{0}-1}^{2}} \cup \overline{e_{l_{0}}^{2}}\right) \\
& X_{3}=\overline{e^{3}} \cup X_{2}
\end{aligned}
$$

Define

$$
U^{4}=P-\left\{E_{v_{1}} \cup \ldots \cup E_{v_{l_{0}-2}} \cup \partial P_{1} \cup E_{v_{1} u_{1}}\right\} .
$$

Clearly $U^{4}$ is homeomorphic to $\mathbb{R}_{\geq 0}^{2}$. So

$$
\left(\mathbb{T}^{2} \times U^{4}\right) / \sim \cong B^{4}=\left\{x \in \mathbb{R}^{4}:|x|<1\right\} .
$$

Define

$$
e^{4}=\left(\mathbb{T}^{2} \times U^{4}\right) / \sim \text { and } X_{4}=\overline{e^{4}} .
$$

For the above $C W$ structure, by reasons of either dimension or orientation, the cellular boundary maps $d_{2}, d_{3}, d_{4}$ are zero. Since $X_{1}$ is homotopic to a circle, we get the following result.

Theorem 3.1. Suppose $P$ is a 2-polytope with one hole. Then

$$
H_{i}(M(P, \lambda), \mathbb{Z})= \begin{cases}\mathbb{Z}^{l_{0}+l_{1}} & \text { if } i=2, \\ \mathbb{Z} & \text { if } i=0,1,3,4, \\ 0 & \text { if } i>4 .\end{cases}
$$

We can give a similar $C W$ structure on $M(P, \lambda)$ when $P$ is a 2-polytope with multiple holes. Figure 2(b) gives a representation of the 1-skeleton of such a structure when there are two holes.

Corollary 3.2. Suppose $P$ is a 2-polytope with $m$ vertices and s simple holes. Then

$$
H_{i}(M(P, \lambda), \mathbb{Z})= \begin{cases}\mathbb{Z}^{m+2 s-2} & \text { if } i=2, \\ \mathbb{Z}^{s} & \text { if } i=1,3, \\ \mathbb{Z} & \text { if } i=0,4, \\ 0 & \text { if } i>4 .\end{cases}
$$




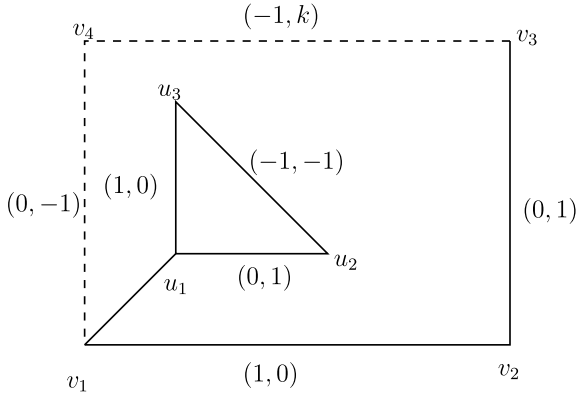

(a)

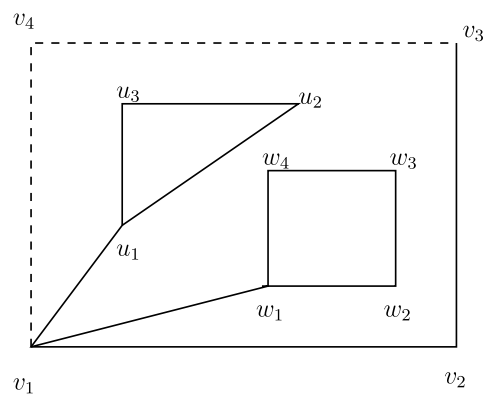

(b)

FIgURE 2. 1-skeleta for 2-polytopes with (a) one hole and (b) two holes.

3.1. Cohomology ring. Assume that $M$ has the characteristic omniorientation. In dimension four it is possible to compute the cohomology ring by using Poincaré duality and intersection product. To illustrate, we consider the case when there is one hole. Let $x_{k} \in H_{2}(M)$ denote the homology class of the sphere associated to the 2 -cell $e_{k}^{2}$. Here characteristic orientation is chosen for the sphere if $k \neq l_{0}-1, l_{0}$. Otherwise orientation determined by the direction $v_{1} u_{1}$ and standard orientation of the associated $S^{1}$ is assumed.

The products of two classes $x_{i}$ and $x_{j}$, when $i$ and $j$ are both less than $l_{0}-1$, is the same as obtained by considering them as classes in $H_{*}\left(M_{0}\right)$. This is because the homotopies needed to achieve transversality can be done away from a neighborhood of any given principal torus fiber. Similar remarks apply when $i$ and $j$ both exceed $l_{0}$. If $i<l_{0}-1$ and $j>l_{0}$, or vice versa, then the product is obviously zero.

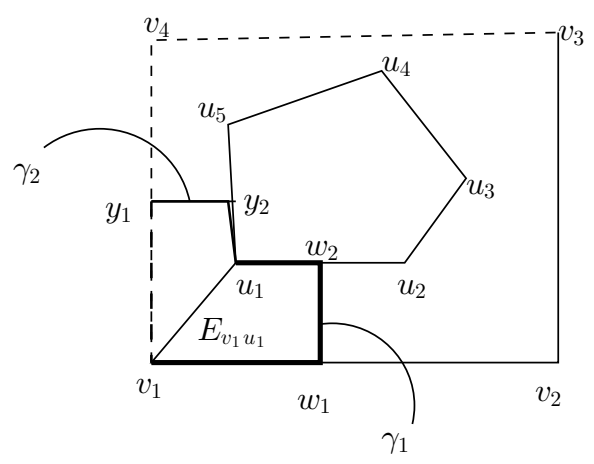

Figure 3 . Homotopic copies of $x_{l_{0}-1}$, here $l_{0}=5$ and $l_{1}=4$.

Now consider the class $x_{l_{0}-1}$. To compute the self intersection $x_{l_{0}-1}^{2}$, we choose two different homotopy representatives, $S_{1}^{2}$ and $S_{2}^{2}$, of $x_{l_{0}-1}$ which intersect only at $v_{1}$ and $u_{1}$. Let $w_{1}, y_{1}$ be points in the relative interior of the edges $v_{1} v_{2}$ and $v_{l_{0}} v_{1}$ respectively. Similarly, let $w_{2}, y_{2}$ be points in the relative interior of the edges $u_{1} u_{2}$ and $u_{l_{1}} u_{1}$ respectively. Let $\gamma_{1}, \gamma_{2}$ be the piecewise linear paths $v_{1} w_{1} w_{2} u_{1}$ and $v_{1} y_{1} y_{2} u_{1}$ respectively. Let $S_{i}^{2}$ be the homotopy sphere $\left(\{1\} \times S^{1}\right) \times \gamma_{i} / \sim$. The circle subgroup $\{1\} \times S^{1}$ corresponds to the submodule of $\mathbb{Z}^{2}$ generated by $(0,1)$. It is possible to express $(0,1)$ uniquely as an integral linear combination 
$a_{1} \lambda_{1}+a_{2} \lambda_{l_{0}}$. Let $d=\operatorname{det}\left[\lambda_{l_{0}}, \lambda_{1}\right]=\sigma\left(v_{1}\right)$. Near $v_{1}$, the sphere $S_{1}^{2}$ is homotopic to $a_{2} d$ times the characteristic sphere over $v_{1} v_{2}$. Similarly, $S_{2}^{2}$ is homotopic to $a_{1} d$ times the characteristic sphere over $v_{1} v_{l_{0}}$. Therefore the contribution of $v_{1}$ to $x_{l_{0}-1}^{2}$ is $(d)\left(a_{1} d\right)\left(a_{2} d\right)=a_{1} a_{2} d$; see Remark 2.5. The contribution from the point $u_{1}$ may be calculated similarly. Other intersection products of degree 2 classes may be calculated by using similar homotopies. For example, $x_{1} \cdot x_{l_{0}-1}=(d)\left(a_{1} d\right)=a_{1}$. Finally the intersection of the generators degree one and degree three homology classes is 1 up to sign.

Example 3.3. Consider $M$ to be the fiber sum of a Hirzebruch surface with $\mathbb{C P}^{2}$ corresponding to Figure 2(a). Let $x_{1}, \ldots, x_{7}$ be the generators of $H_{2}(M)$ as defined above. Let $y$ and $z$ be the generators of $H_{1}(M)$ and $H_{3}(M)$ corresponding to the cells $\sum_{j=4}^{6} e_{j}^{1}$ and $e^{3}$ respectively. Then

$$
\begin{aligned}
& x_{1}^{2}=x_{3}^{2}=x_{4}^{2}=0, x_{2}^{2}=-k, x_{i}^{2}=1 \text { if } i \geq 5, \\
& x_{1} x_{3}=x_{2} x_{3}=x_{2} x_{4}=x_{3} x_{6}=x_{3} x_{7}=x_{4} x_{5}=x_{4} x_{6}=0, \\
& x_{i} x_{j}=0 \text { if } i=1,2 \text { and } j=5,6,7, \\
& x_{1} x_{2}=x_{1} x_{4}=x_{5} x_{6}=x_{5} x_{7}=x_{6} x_{7}=1, x_{3} x_{5}=x_{4} x_{7}=-1, y z=1 .
\end{aligned}
$$

\section{Moment Angle MANifold}

Let $M$ be the manifold obtained by fiber summing the smooth quasitoric manifolds $M_{i}\left(P_{i}, \lambda_{i}\right)$. We may assume that each $P_{i}$ lies in a distinct copy of $\mathbb{R}^{n}$. Let $S_{i}$ be the one point compactification of the copy of $\mathbb{R}^{n}$ that contains $P_{i}$, with standard smooth structure.

The orbit space $O$ of $M$ inherits a smooth structure from the gluing operations in Lemma 2.1, As noted in Remark 2.1, $O$ is homeomorphic to $P$. Using the punctured balls $B_{k}^{-}$as tubes between different affine copies of $\mathbb{R}^{n}$, we may construct a smooth embedding of $O$ into $\mathbb{R}^{n+s}$, where $s$ is the number of holes of $P$. However, we need more. Consider the manifold with corners $\mathrm{O}^{+}$, obtained by gluing a punctured copy of each $S_{i}, 1 \leq i \leq s$, to $P_{0}$ punctured at $s$ points, according to the gluing maps in Lemma 2.1. Then $O^{+}$is homeomorphic to $P_{0}$. We may smoothly embed $O^{+}$into $\mathbb{R}^{n+s}$

For notational simplicity we will describe the embedding in terms of $P$ and the $P_{i}$ s. Induce smooth structures on $P$ and $P_{0}$ using the homeomorphisms with $O$ and $O^{+}$respectively. Then there exists a smooth embedding $\psi_{0}$ of $P_{0}$ in $\mathbb{R}^{n+s}=$ $\left\{\left(p_{1}, \ldots, p_{n+s}\right)\right\}$ such that the following hold:

(1) The image of $P-\bigcup_{k=1}^{s} V_{k}$, where $V_{k}$ is a small neighborhood of $P_{k}$ in $\mathbb{R}^{n}$, lies in $\mathbb{R}^{n}=\left\{p_{1}, \ldots, p_{n}\right\}$.

(2) The image of $V_{k}$ lies in the $(n+1)$-dimensional subspace $\left\{p_{n+j}=0 \mid 1 \leq\right.$ $j \neq k \leq s\}$. The projection of $\psi_{0}\left(V_{k}\right)$ to $\mathbb{R}^{n}$ lies inside $V_{k}$.

(3) The embedding $\psi_{0}$ is affine when restricted to the boundary $\partial P_{k}$. The image of $\partial P_{k}$ lies in the affine subspace $H_{k}:=\left\{p_{n+k}=1, p_{n+j}=0 \forall j\right.$ such that $1 \leq j \neq k \leq s\}$.

(4) $\psi_{0}\left(P_{0}\right) \cap H_{K}=\partial P_{k}$.

(5) The image of $P$ lies between the affine subspaces $p_{n+k}=0$ and $p_{n+k}=1$ for each $1 \leq k \leq s$.

Consider any facet $F_{i}$ of $P$. Suppose $F_{i} \subset P_{k}$ where $k \geq 1$. Choose a linear polynomial $A_{i}$ in the variables $p_{1}, \ldots, p_{n}, p_{n+k}$, other than $a_{k}:=1-p_{n+k}$, which is zero on $\psi_{0}\left(F_{i}\right)$ and positive on $\psi_{0}(P)-\psi_{0}\left(F_{i}\right)$. Define $d_{i}=A_{i}+a_{k}+\sum_{1 \leq j \neq k} p_{n+j}$. 
If $F_{i}$ is a facet of $P_{0}$, then let $A_{i}$ be the defining linear polynomial of $F_{i}$ in the variables $p_{1}, \ldots, p_{n}$ such that $A_{i}$ is positive in the interior of $P_{0}$. In this case define $d_{i}=A_{i}+\sum_{1 \leq j} p_{n+j}$.

Then for a point $x$ in $\psi_{0}(P), d_{i}(x)$ can be thought of as an $l_{1}$-distance of $x$ from the affine subspace of $\psi_{0}\left(F_{i}\right)$. We construct a smooth embedding $\psi_{1}$ of $\psi_{0}\left(P_{0}\right)$ into $\mathbb{R}^{m}$ by $\psi_{1}(x)=\left(d_{1}(x), \ldots, d_{m}(x)\right)$ where $m=|\mathcal{F}(P)|$. The composition $\psi:=\psi_{1} \circ \psi_{0}$ defines an embedding of $P_{0}$ into $\mathbb{R}^{m}=\left\{\left(r_{1}, \ldots, r_{m}\right)\right\}$ such that the image of $P$ lies in $\mathbb{R}_{\geq}^{m}=\left\{r_{i} \geq 0 \forall i\right\}$. Suppose $y \in \psi(P)$. Then $r_{i}(y)=0$ if and only if $y \in \psi\left(F_{i}\right)$.

The space $\mathbb{C}^{m}$ can be regarded as a quotient of $\mathbb{T}^{m} \times \mathbb{R}_{>}^{m}$ by an equivalence relation $\sim_{0}$ as follows: Let $u_{1}, \ldots, u_{m}$ denote the standard basis of $\mathbb{Z}^{m}$. Let $T_{i}$ denote the circle subgroup $\left(\mathbb{Z} u_{i} \otimes \mathbb{R}\right) / \mathbb{Z} u_{i}$ of $\mathbb{T}^{m}$. For any face $F=\left\{r_{j}=0 \mid j \in J\right\}$ of $\mathbb{R}_{\geq}^{m}$, we define the subgroup $T_{F}:=\prod_{j \in J} T_{j}$. For any $y$ in $\mathbb{R}_{\geq}^{m}$, let $F(y)$ denote the unique face of $\mathbb{R}_{\geq}^{m}$ whose relative interior contains $y$. Then define $\sim_{0}$ by

$$
(t, x) \sim_{0}(u, y) \text { if } x=y \text { and } u^{-1} t \in T_{F(y)} .
$$

Definition 4.1. Let $\pi_{0}: \mathbb{C}^{m} \rightarrow \mathbb{R}^{m}$ denote the quotient map. Define the moment angle complex $Z(P)$ of $P$ by

$$
Z(P)=\pi_{0}^{-1}(\psi(P))
$$

We may identify $\pi_{0}$ with the smooth map defined coordinate-wise by $z_{i} \mapsto\left|z_{i}\right|^{2}$. This shows that $Z(P)$ is smooth. The details are straightforward and left to the reader.

Given a characteristic function $\lambda$ for $P$, let $\Lambda: \mathbb{Z}^{m} \rightarrow \mathbb{Z}^{n}$ be the linear map defined by $\Lambda\left(u_{i}\right)=\lambda_{i}$. Let $K=\operatorname{ker} \Lambda$ and $T_{K}=(K \otimes \mathbb{R}) / K$. Then it is easy to observe that topologically $Z(P)$ is a principal $T_{K}$ bundle over $M(P, \lambda)$.

The leaf space $\mathcal{M}(P, \lambda)$ of the foliation corresponding to the smooth and free action of $T_{K}$ on $Z(P)$ has a natural smooth structure. Since $\mathbb{T}^{m} \cong T_{K} \times \mathbb{T}^{n}$, it is not hard to check that $\mathcal{M}(P, \lambda)$ supports a smooth action of $\mathbb{T}^{n}$. Moreover $\mathcal{M}(P, \lambda)$ is equivariantly homeomorphic to $M(P, \lambda)$ with respect to this action. There is a one-to-one correspondence between normal orbit types and, in fact, an isomorphism of $\mathbb{T}^{n}$-normal systems (see Dav78]) of $\mathcal{M}(P, \lambda)$ and $M(P, \lambda)$. (Here the smooth structures on the orbit spaces match that of $O$.) All of these may be ascertained by studying the local representations of $Z(P)$, up to equivariant diffeomorphism, by $T_{K} \times \mathbb{C}^{k} \times\left(\mathbb{C}^{*}\right)^{n-k}$ near the faces of $P$. Therefore by Theorem 4.3 of [Dav78, $\mathcal{M}(P, \lambda)$ and $M(P, \lambda)$ are equivariantly diffeomorphic. We will henceforth identify $M(P, \lambda)$ with $\mathcal{M}(P, \lambda)$ without additional comments.

\section{Almost complex structure}

In this section we prove three results: i) That every omniorientation of $M$ determines a stable almost complex structure on it, ii) that if $M$ admits a positive omniorientation and $\operatorname{dim}(M)=4$, then there exists an almost complex structure on $M$ which is equivalent to the associated stable complex structure, and iii) that there exists a $\mathbb{T}^{n}$-invariant almost complex structure on $M$ if and only if $M$ has a positive omniorientation. It is not known to us if the invariant almost complex structure is equivalent to the associated stable almost complex structure. 
Lemma 5.1. Every omniorientation of the torus manifold $M(P, \lambda)$ determines a stable almost complex structure on it.

Proof. Let $Q=\psi(P)$ and $Q_{k}=\psi\left(P_{k}\right)$ where $\psi$ is the embedding of $P_{0}$ into $\mathbb{R}^{m}$ constructed in section 4. The normal bundle of $Q_{0}$ in $\mathbb{R}^{m}$ is trivial as $Q_{0}$ is contractible. Therefore the normal bundle $N_{Q}$ of $Q$ is also trivial. We may in fact identify $N_{Q}$ with a tubular neighborhood of $Q$ in $\mathbb{R}_{>}^{m}$ following an idea in [BR01: Identify $N_{Q}$ with $\left\{(x, v) \mid x \in Q, v \in\left(T_{x} Q\right)^{\perp} \subset \mathbb{R}^{m}\right\}$ where $\perp$ denotes orthogonal complement with respect to the dot product. Then define the map $f: N_{Q} \rightarrow \mathbb{R}_{>}^{m}$ by $f(x, v)=\left(e^{v_{1}} x_{1}, \ldots, e^{v_{m}} x_{m}\right)$ where the $x_{i}$ s and $v_{i}$ s denote the coordinates of $x$ and $v$ respectively. Then a careful analysis of the situation shows that $v \cdot D f_{(x, 0)}(v)=\sum_{i=1}^{m} v_{i}^{2} x_{i}$ is positive. This shows that $D f_{x}\left(N_{Q}\right)$ is transversal to $T_{x} Q$. We identify $N_{Q}$ with $\operatorname{Df}\left(N_{Q}\right)$.

Since a tubular neighborhood of $Q$ in $\mathbb{R}_{\geq}^{m}$ pulls back to a tubular neighborhood of $Z(P)$ in $\mathbb{C}^{m}$ under $\pi_{0}$, we may identify the normal bundle $N_{Z}$ of $Z(P)$ in $\mathbb{C}^{m}$ with $\pi_{0}^{*} N_{Q}$. Therefore $N_{Z}$ is trivial. Let $N_{M}$ denote the pullback of $N_{Q}$ to $M$ under $\psi \circ \pi$. Then by a slight generalization of the Atiyah sequence [At57, we obtain the following split exact sequence of bundles:

$$
0 \rightarrow \mathfrak{t}_{K} \times M \rightarrow\left(T Z(P) \oplus N_{Z}\right)^{T_{K}} \rightarrow T M \oplus N_{M} \rightarrow 0
$$

Here $\mathfrak{t}_{K}$ denotes the Lie algebra of $T_{K}$. Since the action of $T_{K}$ on $T \mathbb{C}^{m}$ is complex linear, therefore $\left(T Z(P) \oplus N_{Z}\right)^{T_{K}}=\left(\left.T \mathbb{C}^{m}\right|_{Z(P)}\right)^{T_{K}}$ inherits a complex structure. It follows that $M$ admits a stable almost complex structure.

As $T_{K}$ acts diagonally on $\mathbb{C}^{m}$, the bundle $\left(\left.T \mathbb{C}^{m}\right|_{Z(P)}\right)^{T_{K}}$ splits naturally into a direct sum of $m$ complex line bundles over $M$, namely $\nu_{1}, \ldots, \nu_{m}$, corresponding to the complex coordinate directions of $\mathbb{C}^{m}$. These directions correspond to (distance from) the facets of $P$. Since the angular direction $u_{i}$ maps to $\lambda_{i}$ by $\Lambda$, the bundle $\nu_{i}$ restricts to the normal bundle of $X_{i}$ on the characteristic submanifold $X_{i}$. The total Chern class of $M(P, \lambda)$ associated to the above stable complex structure admits the following product decomposition:

$$
c(T M)=\prod_{i=1}^{m}\left(1+c_{1}\left(\nu_{i}\right)\right) .
$$

Using standard localization formula or Theorem 5.3 we obtain

$$
c_{n}(T M)=\sum \sigma(v)
$$

where the sum is over all vertices of $P$.

Lemma 5.2. If $M(P, \lambda)$ admits a positive orientation and $\operatorname{dim}(M)=4$, then it admits an almost complex structure which is equivalent to the associated stable almost complex structure.

Proof. By Theorem 1.7 of [Tho67, the lemma holds if $c_{2}(T M)=e(T M)$. This follows from (5.3) and Corollary 3.2 .

Theorem 5.1. The torus manifold $M(P, \lambda)$ admits a $\mathbb{T}^{n}$-invariant almost complex structure if and only if it has a positive omniorientation.

Proof. The necessity of positive omniorientation for existence of $\mathbb{T}^{n}$-invariant almost complex structure follows from a similar argument as in a quasitoric case; see BP02. 
To prove sufficiency, first assume that the number of holes is one. Note that a positive omniorientation of $M(P, \lambda)$ induces positive omniorientation on $M_{0}$ and $M_{1}$. Then by the work of Kustarev [Kus09], there exist $\mathbb{T}^{n}$-invariant orthogonal almost complex structures $J_{k}$ on $M_{k}, k=0,1$. In particular, these structures are orientation preserving. We may assume that the complex structure $J_{k}$ is locally constant in the normal direction near $L_{k}$, as explained below.

Recall the orientation preserving diffeomorphisms $f_{k}$ in (2.7). Since $T\left(\mathbb{T}^{n} \times B\right)$ is trivial, $d f_{k}$ defines an isomorphism

$$
d f_{k}: T U_{k} \rightarrow \mathbb{T}^{n} \times B \times \mathbb{R}^{2 n} .
$$

Consider the almost complex structures

$$
\widehat{J}_{k}=d f_{k} \circ J_{k} \circ d f_{k}^{-1}
$$

on $\mathbb{T}^{n} \times B \times \mathbb{R}^{2 n}$. Choose a smooth nondecreasing function $\gamma: \mathbb{R} \rightarrow \mathbb{R}$ such that

$$
\gamma(t)= \begin{cases}0 & \text { if } t \leq \epsilon_{1} \\ t & \text { if } t \geq \epsilon_{2}\end{cases}
$$

where $0<\epsilon_{1}<\epsilon_{2}<1$ are small real numbers. Define

$$
J_{k}^{\prime}(\mathbf{q}, r, \Theta)=\widehat{J}_{k}(\mathbf{q}, \gamma(r), \Theta) .
$$

Replace $J_{k}$ by $d f_{k}^{-1} J_{k}^{\prime} d f_{k}$ on $U_{k}$. Denote the resulting almost complex structure on $M_{k}$ by $J_{k}$ without confusion. Note that these new almost complex structures are orientation preserving and $\mathbb{T}^{n}$-invariant.

Recall the orientation preserving diffeomorphism $g$ in (2.9). Define

$$
\phi_{0}:=f_{0}, \quad \phi_{1}:=(I d \times g) \circ f_{1}: U_{1}-L_{1} \rightarrow \mathbb{T}^{n} \times B^{-} .
$$

We have orientation preserving isomorphisms,

$$
d \phi_{k}: T\left(U_{k}-L_{k}\right) \rightarrow \mathbb{T}^{n} \times B^{-} \times \mathbb{R}^{2 n} .
$$

Consider the almost complex structures

$$
\widetilde{J}_{k}=d \phi_{k} \circ J_{k} \circ d \phi_{k}^{-1}
$$

on $\mathbb{T}^{n} \times B^{-} \times \mathbb{R}^{2 n}$. The space of orientation preserving almost complex structures on $\mathbb{R}^{2 n}$ may be identified with $G L^{+}(2 n, \mathbb{R}) / G L(n, \mathbb{C})$. Since $\phi_{k}$ is orientation preserving, we can regard $\widetilde{J}_{k}$ as a map

$$
\widetilde{J}_{k}: \mathbb{T}^{n} \times B^{-} \rightarrow G L^{+}(2 n, \mathbb{R}) / G L(n, \mathbb{C}) .
$$

Since $J_{k}$ is locally constant in the normal direction near $L_{k}$, we may define

$$
\widetilde{J}_{0}(\mathbf{q}, 0, \Theta)=\widetilde{J}_{0}\left(\mathbf{q}, \epsilon_{1} / 2, \Theta\right), \quad \widetilde{J}_{1}(\mathbf{q}, 1, \Theta):=\widetilde{J}\left(\mathbf{q}, 1-\epsilon_{1} / 2, \Theta\right) .
$$

The space $G L^{+}(2 n, \mathbb{R}) / G L(n, \mathbb{C})$ is path connected. Hence there exists a smooth path

$F(t):[0.4,0.6] \rightarrow G L^{+}(2 n, \mathbb{R}) / G L(n, \mathbb{C}), F(0.4)=\widetilde{J}_{1}(\mathbf{1}, 1, \Theta), F(0.6)=\widetilde{J}_{0}(\mathbf{1}, 0, \Theta)$.

By $\mathbb{T}^{n}$-invariance, we construct a smooth family of paths $F(\mathbf{q}, t): \mathbb{T}^{n} \times[0.4,0.6] \rightarrow$ $G L^{+}(2 n, \mathbb{R}) / G L(n, \mathbb{C})$,

$$
F(\mathbf{q}, t):=d \mathbf{q} F(t) d \mathbf{q}^{-1},
$$

satisfying $F(\mathbf{q}, 0.4)=\widetilde{J}_{1}(\mathbf{q}, 1, \Theta), F(\mathbf{q}, 0.6)=\widetilde{J}_{0}(\mathbf{q}, 0, \Theta)$. 
Choose a smooth nondecreasing function $\alpha:(0,1) \rightarrow[0,1)$ such that

$$
\alpha(t)= \begin{cases}t & \text { if } t \geq 0.8 \\ 0 & \text { if } t \leq 0.6\end{cases}
$$

Choose another smooth nondecreasing function $\beta:(0,1) \rightarrow(0,1]$ such that

$$
\beta(t)= \begin{cases}t & \text { if } t \leq 0.2 \\ 1 & \text { if } t \geq 0.4\end{cases}
$$

Define a map $\widetilde{J}: \mathbb{T}^{n} \times B^{-} \rightarrow G L^{+}(2 n, \mathbb{R}) / G L(n, \mathbb{C})$ by

$$
\widetilde{J}(\mathbf{q}, r, \Theta)= \begin{cases}\widetilde{J}_{0}(\mathbf{q}, \alpha(r), \Theta) & \text { if } r>0.6 \\ F(\mathbf{q}, r) & 0.6 \geq r \geq 0.4 \\ \widetilde{J}_{1}(\mathbf{q}, \beta(r), \Theta) & \text { if } r<0.4\end{cases}
$$

Note that

$$
\widetilde{J}(\mathbf{q}, r, \Theta)= \begin{cases}\widetilde{J}_{0}(\mathbf{q}, r, \Theta) & \text { if } r>0.8 \\ \widetilde{J}_{1}(\mathbf{q}, r, \Theta) & \text { if } r<0.2 .\end{cases}
$$

Define a $\mathbb{T}^{n}$-invariant almost complex structure $\bar{J}_{k}$ on $T\left(U_{k}-L_{k}\right)$ by

$$
\bar{J}_{k}=d \phi_{k}^{-1} \circ \widetilde{J} \circ d \phi_{k} .
$$

By construction, $\bar{J}_{k}$ agrees with $J_{k}$ in a neighborhood of the outer boundary of $U_{k}-L_{k}$. Therefore $\bar{J}_{k}$ extends to a $\mathbb{T}^{n}$-invariant almost complex structure on $M_{k}-L_{k}$. Moreover $\bar{J}_{0} \circ d h=d h \circ \bar{J}_{1}$ on $U_{1}-L_{1}$ since $h=\phi_{0}^{-1} \circ \phi_{1}$; see (2.10) and (5.8). Therefore $\bar{J}_{0}$ and $\bar{J}_{1}$ glue to produce a $\mathbb{T}^{n}$-invariant almost complex structure $\bar{J}$ on $M$. Finally, note that we may apply induction when the number of holes is greater than one.

Remark 5.2. In dimension four, the sufficiency part of Theorem 5.1 also follows from section 13 of [GK98] together with the main theorem of Kus09].

5.1. The $\chi_{y}$ genus. The Hirzebruch $\chi_{y}$ genus is an invariant of the complex cobordism class of the manifold and thus depends on the stable almost complex structure. We give a combinatorial formula of the $\chi_{y}$ genus of $M$, following Panov's work on quasitoric manifolds. The proofs are the same as in Pan01.

Let $E$ be an edge of $P^{n}$. The isotropy subgroup of $\pi^{-1}(E)$ is an $(n-1)$ dimensional torus generated by a submodule $K$ of rank $(n-1)$ in $\mathbb{Z}^{n}$. A primitive vector $\mu$ in $\left(\mathbb{Z}^{n}\right)^{*}$ is called an edge vector corresponding to $E$ if $\mu(\alpha)=0$ for each $\alpha \in K$. The edge vector of $E$ is therefore unique up to sign.

Let $\nu$ be a primitive vector in $\mathbb{Z}^{n}$ such that

$$
\mu(\nu) \neq 0 \text { for any edge vector } \mu \text {. }
$$

Then the circle $S_{\nu}^{1}=(\mathbb{Z}\langle\nu\rangle \otimes \mathbb{R}) / \mathbb{Z}\langle\nu\rangle$ acts smoothly on $M$ with only isolated fixed points corresponding to the vertices of $P$.

We choose signs for each edge vector at a vertex $v$ according to the characteristic omniorientation as follows. Order the codimension one faces meeting at $v$ and corresponding edges $E_{k} \mathrm{~s}$ as in subsection 2.4. Let $\mu_{k}$ be an edge vector corresponding to $E_{k}$. Let $M_{(v)}$ be the matrix, $M_{(v)}=\left[\mu_{1}, \ldots, \mu_{k}\right]$. Then choose sign for each $\mu_{k}$ such that $M_{(v)}^{t} \Lambda_{(v)}=I_{n}$. Under this choice of signs the action of $S_{\nu}^{1}$ induces a representation of $S^{1}$ on the tangent space $T_{v} M$ with weights $\mu_{1}(\nu), \ldots, \mu_{n}(\nu)$. 
Definition 5.1. Define the index of a vertex $v \in P$ as the number of negative weights of the $S^{1}$ representation on $T_{v}(M)$,

$$
\operatorname{ind}_{\nu}(v)=\left|\left\{k: \mu_{k}(\nu)<0\right\}\right| .
$$

Theorem 5.3. For any vector $\nu$ satisfying (5.20),

$$
\chi_{y}(M)=\sum_{v}(-y)^{\operatorname{ind}_{\nu}(v)} \sigma(v) .
$$

Note that Theorem 5.3 also follows from Lemma 5.1 together with Theorem 10.1 of HM03. Specializing the formula in Theorem 5.3 to $y=-1$ and $y=1$, respectively yield formulas for the top Chern number and the signature. Moreover following Theorem 3.4 of [Pan01] or Theorem 4.2 of [Mas99] we obtain the following formula for Todd genus of $M$ :

$$
\operatorname{td}(M)=\sum_{\operatorname{ind}_{\nu}(v)=0} \sigma(v) .
$$

\subsection{Integrability questions.}

Lemma 5.3. If the polytope $P$ has at least one hole, then the torus manifold $M(P, \lambda)$ does not support any symplectic form for which the torus action is symplectic.

Proof. When the dimension $2 n>4, M(P, \lambda)$ is simply connected. So any symplectic circle action is Hamiltonian. Therefore if $M(P, \lambda)$ supports a $T^{n}$-invariant symplectic form, then the action of $T^{n}$ must be Hamiltonian. Then $M(P, \lambda)$ would be a symplectic toric manifold with a moment map whose image is a Delzant polytope. Then the orbit space of the $T^{n}$-action on $M(P, \lambda)$ would be a Delzant polytope; see Theorem 2.6.2 of [Sil01]. Therefore, as the orbit space of $M(P, \lambda)$ is not convex, it cannot support an invariant symplectic form.

When $2 n=4$, a result of McDuff [McD88] states that a symplectic circle action on a compact four dimensional manifold is Hamiltonian if and only if it has fixed points. Therefore, again, if $M(P, \lambda)$ supports a $T^{n}$-invariant symplectic form, then the action of $T^{n}$ must be Hamiltonian. We get a contradiction as above.

It follows from the main result of IK12 that $M(P, \lambda)$ cannot admit a complex structure with respect to which the torus action is holomorphic if it is not a toric variety, for instance when $P$ has at least one hole.

More generally, we may ask whether $M(P, \lambda)$ admits any symplectic or complex structure. We do not know of any example that does so in case $P$ has at least one hole.

Lemma 5.4. If $P$ is a 2-polytope with an odd number of holes, then $M(P, \lambda)$ can not be Kahler.

Proof. If $P$ has $s$ holes, by Corollary 3.2, the first Betti number $b_{1}(M)=s$. But for a compact Kahler manifold, the Betti numbers of odd degree are even (see GH94, page 117). The result follows.

It is not hard to produce examples of $M$ that admit almost complex structure but do not admit an integrable complex structure. For an almost complex 4-manifold, $c_{1}^{2}$ and $c_{2}$ are determined by the Euler characteristic and signature, and are therefore independent of the choice of almost complex structure. Consider the equivariant 
connected sum $Y$ of three copies of $\mathbb{C} P^{2}$. This is a quasitoric manifold with a pentagon as $P$. The characteristic vectors may be chosen to be $(1,0),(-1,1)$, $(1,-2),(0,1)$ and $(-1,-1)$, thus endowing $Y$ with a positive omniorientation and an almost complex structure. However, $Y$ has $c_{1}^{2}=19$ and $c_{2}=5$. Therefore the Bogomolov-Miyaoka-Yau inequality, $c_{1}^{2} \leq 3 c_{2}$, is not satisfied and $Y$ does not admit a complex structure. It may be argued using (5.2) and intersection theory that $c_{1}^{2}$ and $c_{2}$ are additive with respect to the fiber sum operation. Therefore the fiber sum of any finite number of copies of $Y$ produces an almost complex manifold which does not admit a complex structure. Since $c_{1}^{2}=3 c_{2}$ for $\mathbb{C} P^{2}$, the fiber sum of copies of $Y$ and $\mathbb{C} P^{2}$ also yields such examples.

\section{ACKNOWLEDGEMEnTS}

It is a pleasure to thank Shengda $\mathrm{Hu}$ for extensive discussions. The authors also thank Andres Angel, Saibal Ganguli, Mikhail Malakhaltsev, Taras Panov and Dong Youp Suh for helpful conversations. The authors also thank Mikiya Masuda for comments that helped them improve their exposition. The authors thank Yael Karshon and the referee for pointing out two different serious errors in earlier drafts of the article. The authors also thank the referee for suggesting numerous improvements.

\section{REFERENCES}

[At57] M. F. Atiyah, Complex analytic connections in fibre bundles, Trans. Amer. Math. Soc. 85 (1957), 181-207. MR0086359(19,172c)

[BP02] Victor M. Buchstaber and Taras E. Panov, Torus actions and their applications in topology and combinatorics, University Lecture Series, vol. 24, American Mathematical Society, Providence, RI, 2002. MR1897064(2003e:57039)

[BPV84] W. Barth, C. Peters, and A. Van de Ven, Compact complex surfaces, Ergebnisse der Mathematik und ihrer Grenzgebiete (3) [Results in Mathematics and Related Areas (3)], vol. 4, Springer-Verlag, Berlin, 1984. MR749574 (86c:32026)

[BR01] Victor M. Buchstaber and Nigel Ray, Tangential structures on toric manifolds, and connected sums of polytopes, Internat. Math. Res. Notices 4 (2001), 193-219, DOI 10.1155/S1073792801000125. MR1813798(2002b:57043)

[Dav78] Michael Davis, Smooth G-manifolds as collections of fiber bundles, Pacific J. Math. 77 (1978), no. 2, 315-363. MR510928(80b:57034)

[DJ91] Michael W. Davis and Tadeusz Januszkiewicz, Convex polytopes, Coxeter orbifolds and torus actions, Duke Math. J. 62 (1991), no. 2, 417-451, DOI 10.1215/S0012-7094-9106217-4. MR:1104531 (92i:52012)

[GH94] Phillip Griffiths and Joseph Harris, Principles of algebraic geometry, Wiley Classics Library, John Wiley \& Sons, Inc., New York, 1994. Reprint of the 1978 original. MR 1288523 (95d:14001)

[GK98] Michael D. Grossberg and Yael Karshon, Equivariant index and the moment map for completely integrable torus actions, Adv. Math. 133 (1998), no. 2, 185-223, DOI 10.1006/aima.1997.1686. MR 1604738(2000f:53112)

[Gom95] Robert E. Gompf, A new construction of symplectic manifolds, Ann. of Math. (2) 142 (1995), no. 3, 527-595, DOI 10.2307/2118554. MR1356781(96j:57025)

[Gro86] Mikhael Gromov, Partial differential relations, Ergebnisse der Mathematik und ihrer Grenzgebiete (3) [Results in Mathematics and Related Areas (3)], vol. 9, Springer-Verlag, Berlin, 1986. MR864505 (90a:58201)

[HM03] Akio Hattori and Mikiya Masuda, Theory of multi-fans, Osaka J. Math. 40 (2003), no. 1, 1-68. MR1955796 (2004d:53103)

[IK12] Hiroaki Ishida and Yael Karshon, Completely integrable torus actions on complex manifolds with fixed points, Math. Res. Lett. 19 (2012), no. 6, 1283-1295, DOI 10.4310/MRL.2012.v19.n6.a9. MR3091608 
[Kus09] A. A. Kustarev, Equivariant almost complex structures on quasitoric manifolds (Russian, with Russian summary), Tr. Mat. Inst. Steklova 266 (2009), no. Geometriya, Topologiya i Matematicheskaya Fizika. II, 140-148, DOI 10.1134/S0081543809030080; English transl., Proc. Steklov Inst. Math. 266 (2009), no. 1, 133-141. MR2603265 (2011b:57031)

[McD88] Dusa McDuff, The moment map for circle actions on symplectic manifolds, J. Geom. Phys. 5 (1988), no. 2, 149-160, DOI 10.1016/0393-0440(88)90001-0. MR.1029424 (91c:58042)

[Mas99] Mikiya Masuda, Unitary toric manifolds, multi-fans and equivariant index, Tohoku Math. J. (2) 51 (1999), no. 2, 237-265, DOI 10.2748/tmj/1178224815. MR.1689995 (2000e:57058)

[MP06] Mikiya Masuda and Taras Panov, On the cohomology of torus manifolds, Osaka J. Math. 43 (2006), no. 3, 711-746. MR2283418 (2007j:57039)

[Pan01] T. E. Panov, Hirzebruch genera of manifolds with torus action (Russian, with Russian summary), Izv. Ross. Akad. Nauk Ser. Mat. 65 (2001), no. 3, 123-138, DOI 10.1070/IM2001v065n03ABEH000338; English transl., Izv. Math. 65 (2001), no. 3, 543556. MR1853368 (2002i:57047)

[Sil01] Ana Cannas da Silva, Symplectic toric manifolds, Symplectic geometry of integrable Hamiltonian systems (Barcelona, 2001), Adv. Courses Math. CRM Barcelona, Birkhäuser, Basel, 2003, pp. 85-173. MR2000746

[Tho67] Emery Thomas, Complex structures on real vector bundles, Amer. J. Math. 89 (1967), 887-908. MR0220310(36 \#3375)

Departamento de Matemáticas, Universidad de los Andes, Bogota, Colombia

E-mail address: mainakp@gmail.com

Department of Mathematics, Korea Advanced Institute of Science and Technology, DAeJeOn, Republic OF Korea

E-mail address: soumensarkar20@gmail.com 